\title{
Expert systems for fetal assessment in labour (Protocol)
}

\author{
Lutomski JE, Meaney S, Greene RA, Ryan AC, Devane D
}

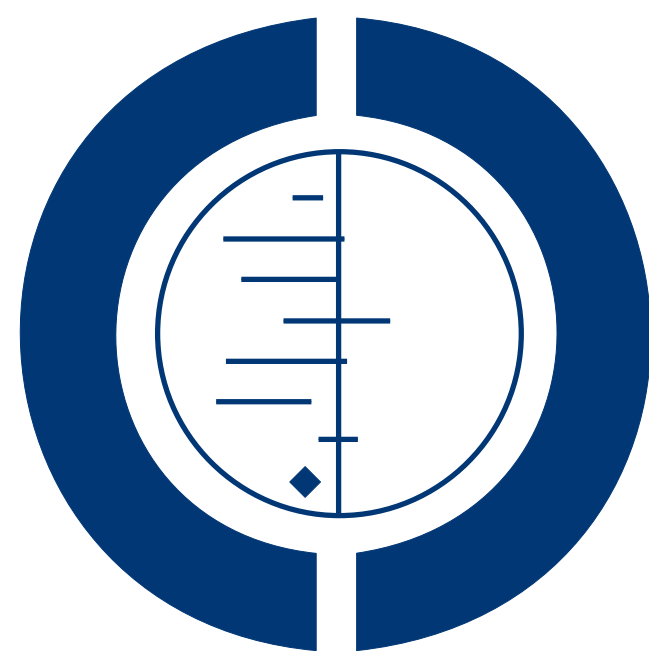

\section{THE COCHRANE COLLABORATION ${ }^{\circledR}$}

This is a reprint of a Cochrane protocol, prepared and maintained by The Cochrane Collaboration and published in The Cochrane Library 2013, Issue 8

http://www.thecochranelibrary.com

\section{WILEY}

Expert systems for fetal assessment in labour (Protocol)

Copyright $\odot 2013$ The Cochrane Collaboration. Published by John Wiley \& Sons, Ltd. 
TABLE OF CONTENTS

HEADER . . . . . . . . . . . . . . . . . . . . . . . . . . . . . . . . . . . . 1

ABSTRACT . . . . . . . . . . . . . . . . . . . . . . . . . . . . . . . . . . . . . . . 1

BACKGROUND . . . . . . . . . . . . . . . . . . . . . . . . . . . . . . . . . . . .

OBJECTIVES . . . . . . . . . . . . . . . . . . . . . . . . . . . . . . . . . . . . . . .

METHODS . . . . . . . . . . . . . . . . . . . . . . . . . . . . . . . . . . . . . .

ACKNOWLEDGEMENTS . . . . . . . . . . . . . . . . . . . . . . . . . . . . . . . . . . . . . . . .

REFERENCES . . . . . . . . . . . . . . . . . . . . . . . . . . . . . . . . . . . . . . 8

APPENDICES . . . . . . . . . . . . . . . . . . . . . . . . . . . . . . . . . . . . . 10

CONTRIBUTIONS OF AUTHORS . . . . . . . . . . . . . . . . . . . . . . . . . . . . . . . . . . . 10

DECLARATIONS OF INTEREST . . . . . . . . . . . . . . . . . . . . . . . . . . . . . . . . . . 10

SOURCES OF SUPPORT . . . . . . . . . . . . . . . . . . . . . . . . . . . . . . . . . 


\title{
[Intervention Protocol]
}

\section{Expert systems for fetal assessment in labour}

\author{
Jennifer E Lutomski ${ }^{1}$, Sarah Meaney ${ }^{1}$, Richard A Greene ${ }^{2}$, Anthony C Ryan ${ }^{3}$, Declan Devane ${ }^{4}$ \\ ${ }^{1}$ National Perinatal Epidemiology Centre, Wilton, Ireland. ${ }^{2}$ Department of Obstetrics and Gynaecology, National Perinatal Epidemiol- \\ ogy Centre, Wilton, Ireland. ${ }^{3}$ Neonatal Intensive Care Unit, Cork University Maternity Hospital, Wilton, Ireland. ${ }^{4}$ School of Nursing \\ and Midwifery, National University of Ireland Galway, Galway, Ireland
}

Contact address: Jennifer E Lutomski, National Perinatal Epidemiology Centre, 5th Floor, Cork University Maternity Hospital, Wilton, Cork, Ireland. j.lutomski@ucc.ie.

Editorial group: Cochrane Pregnancy and Childbirth Group.

Publication status and date: New, published in Issue 8, 2013.

Citation: Lutomski JE, Meaney S, Greene RA, Ryan AC, Devane D. Expert systems for fetal assessment in labour. Cochrane Database of Systematic Reviews 2013, Issue 8. Art. No.: CD010708. DOI: 10.1002/14651858.CD010708.

Copyright (C) 2013 The Cochrane Collaboration. Published by John Wiley \& Sons, Ltd.

\begin{abstract}
A B S T R A C T
This is the protocol for a review and there is no abstract. The objectives are as follows:

To evaluate the effectiveness of continuous or intermittent CTG monitoring during labour with an ES compared with (1) continuous or intermittent CTG monitoring during labour without an ES or (2) intermittent auscultation with a Pinard stethoscope or hand-held Doppler ultrasound device.
\end{abstract}

\section{B A C K G R O U N D}

Intrapartum fetal hypoxia (shortage of oxygen) is a serious complication of labour which increases the risk of perinatal mortality and morbidity, including hypoxic ischaemic encephalopathy (acute or subacute brain injury due to asphyxia), cerebral palsy and developmental delay (Dilenge 2001; Fatemi 2009; McIntyre 2013). Whereas a healthy, term baby has a baseline heart rate between 110 and 160 beats per minute, moderate variations in heart rate amplitude and natural accelerations from its baseline heart rate, this may not be the case for the hypoxic baby (ACOG 2009; Liston 2007; NICE 2007; RANZCOG 2006). In contrast, a hypoxic baby may display minimal or a complete absence of heart rate variation and accelerations as well as have marked decelerations from its baseline heart rate values (ACOG 2009; Liston 2007; NICE 2007; RANZCOG 2006). Since abnormal fetal heart rate patterns may signify poor oxygenation and thus potential fetal compromise, maternal care professionals often perform fetal assessment during labour.
Cardiotocography (CTG, also known as electronic fetal heart rate monitoring) and intermittent auscultation of the fetal heart rate are the most common forms of fetal assessment during labour (ACOG 2009; Liston 2007; NICE 2007; RANZCOG 2006). In brief, CTG records the fetal heart rate and maternal uterine activity via two transducers usually placed on the mother abdomen; an ultrasound transducer measures fetal heart rate and a pressure transducer measures uterine contractions. Fetal heart rate and uterine contractions are printed on a strip known as a CTG trace or presented directly on a visual display unit to help identify abnormal readings. In contrast, intermittent auscultation is a method of listening to the baby's heart rate using a Pinard (fetal stethoscope) or a hand-held Doppler ultrasound device. The rationale underpinning both forms of fetal assessment is that babies who are at risk of becoming compromised can be identified early so that appropriate recourse can be made (i.e. more intensive monitoring or expedited birth of the baby) (ACOG 2009; Liston 2007; NICE 2007; RANZCOG 2006). 


\section{Description of the condition}

In the early half of the twentieth century, intermittent auscultation served as the primary form of fetal assessment (Liston 2007). Technological advancements during this time period led to the development of CTG, and this method of fetal assessment was subsequently introduced into maternity care in the late 1960s (Chez 2011). Despite early beliefs that CTG could potentially supersede intermittent auscultation (Liston 2007), since its induction, there has been a growing professional debate on the efficacy of CTG monitoring (Alfirevic 2013; Chauhan 2008; Devane 2012; Hill 2012; Miller 2011a). Although CTG monitoring is typically reserved for high-risk pregnancies, in the many parts of the developed world, more than three-quarters of babies will be assessed using CTG (ACOG 2009; Devane 2007; Devane 2012). Yet, CTG has a relatively low specificity (a high false positive rate) for identifying fetal hypoxia and associated complications (Miller 2011a). For example, the false positive rate for the most abnormal of fetal heart rate patterns, the detection of cerebral palsy, has been reported as high as $99.8 \%$ (Nelson 1996). This lack of specificity can be attributed, in part, to the variability in interpretation of fetal heart rate traces. Despite guidelines for CTG interpretation, substantial inter- and intra-observer variation in interpretation has been reported among maternity care providers (Blix 2003; Chauhan 2008; Devane 2005; Figueras 2005). Misinterpretation of fetal heart rate traces can lead to poor decisions, which can result in unnecessary intervention or delay or withholding of necessary intervention. Together, this can impact on the risks of operative birth, perinatal asphyxia, hypoxic ischaemic encephalopathy and perinatal death.

Given these clinical implications, substantial research has investigated CTG monitoring in its current form versus alternative methods. In a recent systematic review, women with signs of labour who received a short, 20-minute CTG tracing on admission to the maternity ward were more likely to have a caesarean birth than women who were monitored using intermittent auscultation (risk ratio (RR) 1.20; 95\% confidence interval (CI) 1.00 to 1.44 ) (Devane 2012). A related review found that in comparison to intermittent auscultation, continuous CTG during labour significantly decreased the risk of neonatal seizures (RR 0.50; 95\% CI 0.31 to 0.80 ), though it did not decrease the risk of cerebral palsy (RR 1.75; 95\% CI 0.84 to 3.63) (Alfirevic 2013). Further, continuous CTG was associated with significantly higher rates of caesarean (RR 1.63; 95\% CI 1.29 to 2.07) and instrumental births (RR 1.15; 95\% CI 1.01 to 1.33 ). Thus, while CTG can provide critical information to maternity care providers, it is not without limitations.

\section{Description of the intervention}

First developed in the 1960s, expert systems (ESs) represent a type of applied artificial intelligence designed to assist in complex deci- sion-making (Liao 2005). In a healthcare context, ESs synthesise a computerised knowledge base derived from expert opinion with individual patient data to guide users towards possible diagnosis or treatment decisions (Liao 2005). To process data, an ES may apply rule-based algorithms or neural networks (i.e. a model of pattern recognition based on previously collected data) (McCartney 2000; McCartney 2011); notably, however, there are numerous other ES methodologies (Liao 2005). Requirements for ESs vary; systems may be web-based or supported on a stand alone personal computer. ESs are paperless and often represent real-time, which is critical in healthcare environments where changes in health status can occur rapidly. Advancements in ESs have led to their integration in a wide range of clinical settings, including cardiac care (Chi 2012; Seto 2012), cancer diagnosis (Issac Niwas 2012; Yang 2012) and diabetes diagnosis (Basciftci 2011; Picon 2012).

The potential for ESs in maternity care is also well recognised, and as a result, there has been an increasing interest in developing ESs for CTG monitoring (Ayres-de-Campos 2010; Greene 1996). Such an ES would combine information on maternal and fetal characteristics, which may include fetal heart rate, electrocardiogram (ECG) waveform, uterine contractions and/or gestational age, to provide a comprehensive overview of the labour and/or issue an alert if the baby's status becomes critical. ES-issued alerts may not only advise the user on potentially suitable intervention but also provide underlying reasons for this recommendation, simulating reasoning capacity that can be interpreted easily by the user (Liao 2005).

\section{How the intervention might work}

Despite controversy surrounding its ubiquitous use, CTG monitoring continues to be recommended by a number of professional organisations for the monitoring of at least specific subgroups of pregnant women (Miller 2011a). Yet, there is general consensus that measures must be taken to improve its interpretation (Chez 2011). For this reason, CTG clinical definitions and guidelines have undergone extensive review (ACOG 2009; Liston 2007; NICE 2007; RANZCOG 2006) and training programmes have been recommended and evaluated (Pehrson 2011). Arguably, however, guidelines and educational interventions alone may be insufficient in reducing inter-observer differences (Devoe 2000). Although complementary measurements to enhance CTG interpretation, such as fetal pulse oximetry, lactate level measurements and fetal ECG waveform analysis, have been investigated, to date, these techniques have conferred limited advantages over conventional CTG monitoring (East 2007; East 2010; Neilson 2012). In particular, issues in interpretability were reported for ECG waveform analysis, which may have impacted, in part, its utility as an additional indicator of fetal compromise (Steer 2008). Consequently, conventional CTG monitoring has remained the norm across many maternity units. 
In this context, an ES for fetal assessment represents a potential alternative mechanism to counter issues with observer bias and improve interpretation of fetal heart rate tracings. Developing an ES to improve CTG monitoring has been a goal in maternity care for decades. Whereas earlier versions displayed only limited successes, substantial advances have been made in intelligence software (Devoe 2000; Greene 1996; Guijarro-Berdiñas 2002; Steer 2008). Moreover, several observational studies have reported significantly improved levels of agreement between practitioners interpreting fetal heart rate patterns and identifying adverse outcomes when assisted by an ES (Ayres-de-Campos 2010; Costa 2010; Costa 2010a). Thus, improving diagnostic interpretation may result in improved identification of truly compromised babies who warrant intervention.

\section{Why it is important to do this review}

Given that a high proportion of women will receive a CTG on their admission to the labour ward and/or will be monitored continuously throughout their labour (ACOG 2009; Devane 2012), it is critical to ensure that these CTGs are interpreted to the highest level of accuracy to minimise sub-optimal clinical decisions and outcomes. Improved CTG interpretation has clear short- and long-term health benefits for both mothers and babies through possible prevention of serious neonatal complications and unnecessary operative birth. There are also important economic implications for the healthcare system. Due to long-term care requirements, neonatal cases of hypoxia and cerebral palsy can lead to costly malpractice suits (Miller 2011b); thus, reducing morbidity incidence through enhanced fetal assessment could potentially have profound impact in this regard. Further, reducing unnecessary caesarean birth also benefits the healthcare system. Not only is caesarean birth typically twice the cost of a vaginal birth (Fawsitt 2013; Henderson 2001), but also, particularly in a nulliparous woman, may initiate a legacy of increased health costs (Grobman 2000) as a result of higher risk of subsequent caesarean birth and medical complications (Garmi 2012; Lydon-Rochelle 2010).

\section{O B J E C T I VES}

To evaluate the effectiveness of continuous or intermittent CTG monitoring during labour with an ES compared with (1) continuous or intermittent CTG monitoring during labour without an ES or (2) intermittent auscultation with a Pinard stethoscope or hand-held Doppler ultrasound device.

\section{Criteria for considering studies for this review}

\section{Types of studies}

We will include all randomised, cluster-randomised and quasi-randomised trials comparing continuous or intermittent CTG monitoring during labour with an ES with continuous or intermittent CTG monitoring without an ES. We will also include trials that compare continuous or intermittent CTG monitoring during labour with an ES with intermittent auscultation with a Pinard or hand-held Doppler.

Given the rapidity at which the health status of the mother and baby can change during labour, cross-over trials are unsuitable for the intervention under review and therefore will be excluded.

\section{Types of participants}

Pregnant women in labour and their babies.

\section{Types of interventions}

We will make the following comparisons.

1. Continuous CTG monitoring with an ES versus continuous CTG monitoring alone.

2. Continuous CTG monitoring with an ES versus intermittent CTG monitoring alone.

3. Continuous CTG monitoring with an ES versus intermittent auscultation with a Pinard stethoscope or hand-held Doppler ultrasound device.

4. Intermittent CTG monitoring with an ES versus intermittent CTG monitoring alone.

5. Intermittent CTG monitoring with an ES versus continuous CTG monitoring alone.

6. Intermittent CTG monitoring with an ES versus intermittent auscultation with a Pinard stethoscope or hand-held Doppler ultrasound device.

For the purposes of this review, we define continuous CTG monitoring as monitoring of the fetal heart rate and uterine contractions, which commenced at some point during labour, and upon commencement, was only discontinued for short periods of time. We define intermittent monitoring as monitoring of the fetal heart rate and uterine contractions which were not continuous but rather at select intervals during labour.

We define an ES as any applied artificial intelligence tool designed to assist in complex decision-making by integrating a computerised knowledge base derived from expert opinion with individualised patient data (Liao 2005). Given that the underlying factors built into one ES may differ from others, where sufficient detail is provided by trial authors, we will describe which maternal and baby characteristics are included in the ES.

\section{METHODS}




\section{Types of outcome measures}

Primary outcomes

\section{Maternal}

1. Incidence of caesarean birth.

\section{Baby}

1. Incidence of perinatal mortality defined as fetal deaths (a baby delivered without signs of life at $\geq 22$ weeks' gestation and/ or with a birthweight $\geq 500 \mathrm{~g}$ ) and neonatal deaths (death of a liveborn baby $\geq 22$ weeks' gestation and/or with a birthweight $\geq$ $500 \mathrm{~g}$ occurring within 28 days after birth) excluding lethal congential anomalies.

2. Incidence of neonatal seizures.

3. Acidemia as evidenced by a $\mathrm{pH}$ less than $7.0 \mathrm{and} /$ or a base deficit greater than $12 \mathrm{mmol} / \mathrm{L}$ in umbilical arterial cord blood or neonatal blood sample within the first hour of life, or both.

\section{Secondary outcomes}

\section{Maternal}

1. Incidence of operative vaginal birth (ventouse or forceps).

2. Incidence of fetal blood sampling.

3. Incidence of artificial rupture of amniotic membranes.

4. Incidence of oxytocin augmentation of labour.

\section{Baby}

1. Incidence of hypoxic ischaemic encephalopathy as defined by trial authors.

2. Incidence of admission to neonatal special care and/or neonatal intensive care unit.

3. Apgar score less than seven at five minutes.

\section{Search methods for identification of studies}

\section{Electronic searches}

We will contact the Trials Search Co-ordinator to search the Cochrane Pregnancy and Childbirth Group's Trials Register. The Cochrane Pregnancy and Childbirth Group's Trials Register is maintained by the Trials Search Co-ordinator and contains trials identified from:

1. monthly searches of the Cochrane Central Register of Controlled Trials (CENTRAL);
2. weekly searches of MEDLINE;

3. weekly searches of Embase;

4. handsearches of 30 journals and the proceedings of major conferences;

5. weekly current awareness alerts for a further 44 journals plus monthly BioMed Central email alerts.

Details of the search strategies for CENTRAL, MEDLINE and EMBASE, the list of handsearched journals and conference proceedings, and the list of journals reviewed via the current awareness service can be found in the 'Specialized Register' section within the editorial information about the Cochrane Pregnancy and Childbirth Group.

Trials identified through the searching activities described above are each assigned to a review topic (or topics). The Trials Search Co-ordinator searches the register for each review using the topic list rather than keywords.

For the purposes of this review, we will also include studies published as abstracts if we are able to extract sufficient information on the trial. In cases where insufficient data are published, we will first contact the trial authors to access required information. If after contacting the trial authors data remain insufficient, the abstract will be excluded from our review.

In addition, we plan to search grey literature through Open Grey and ProQuest Dissertation \& Theses Database using the search terms given in Appendix 1

\section{Searching other resources}

We will review citations of reference lists of included papers identified through the above search strategy and assess their suitability for inclusion in the review.

We will not apply any language restrictions.

\section{Data collection and analysis}

The methods that will be carried out in this review were designed in accordance with recommendations described in the Cochrane Handbook for Systematic Reviews of Interventions (Higgins 2011).

\section{Selection of studies}

Two review authors (Jennifer E Lutomski (JEL) and Declan Devane (DD)) will independently assess for inclusion all the potential studies we identify as a result of the search strategy. We will resolve any disagreement through discussion or, if required, we will consult a third review author (CA Ryan (CAR), Sarah Meaney (SM) or Richard A Greene (RAG)).

\section{Data extraction and management}

We will design a form to extract data. For eligible studies, two review authors (JEL and SM) will extract the data using the agreed 
form. We will resolve discrepancies through discussion or, if required, we will consult a third review author (DD). We will enter data into Review Manager software (RevMan 2011) and check for accuracy. When information regarding any of the above is unclear, we will attempt to contact authors of the original reports to provide further details.

\section{Assessment of risk of bias in included studies}

Two review authors (JEL and DD) will independently assess risk of bias for each study using the criteria outlined in the Cochrane Handbook for Systematic Reviews of Interventions (Higgins 2011). We will resolve any disagreement by discussion or by involving a third review author.

\section{(I) Random sequence generation (checking for possible selection bias)}

We will describe for each included study the method used to generate the allocation sequence in sufficient detail to allow an assessment of whether it should produce comparable groups. We will assess the risk of bias for sequence generation as:

- low risk (any truly random process, e.g. random number table; computer random number generator);

- high risk (any non-random process, e.g. odd or even date of birth; hospital or clinic record number); or

- unclear risk.

\section{(2) Allocation concealment (checking for possible selection} bias)

We will describe for each included study the method used to conceal allocation to interventions prior to assignment and will assess whether intervention allocation could have been foreseen in advance of, or during recruitment, or changed after assignment. We will assess the risk of bias for allocation concealment as:

- low risk (e.g. telephone or central randomisation; consecutively numbered sealed opaque envelopes);

- high risk (e.g. open random allocation; unsealed or nonopaque envelopes, alternation; date of birth); or

- unclear risk.

\section{(3.I) Blinding of participants and personnel (checking for possible performance bias)}

Given the additional equipment required to support an ES, it is not possible for personnel to be blinded to the intervention in these trials. However, we will describe the methods used, if any, to blind women from knowledge of the intervention. We will consider that studies are at low risk of bias if women were blinded or if we judge that the lack of blinding would not likely impact results. We will assess the risk of bias for blinding of participants as:

- low risk;
- high risk; or

- unclear risk.

(3.2) Blinding of outcome assessment (checking for possible detection bias)

We will describe for each included study the methods used, if any, to blind outcome assessors from knowledge of which intervention a woman received. We will assess blinding separately for different outcomes or classes of outcomes. We will assess the risk of bias for blinding of outcome assessment as:

- low risk;

- high risk; or

- unclear risk.

(4) Incomplete outcome data (checking for possible attrition bias due to the amount, nature and handling of incomplete outcome data)

We will describe for each included study, and for each outcome or class of outcomes, the completeness of data including attrition and exclusions from the analysis. We will state whether attrition and exclusions were reported and the numbers included in the analysis at each stage (compared with the total randomised participants), reasons for attrition or exclusion where reported, and whether missing data were balanced across groups or were related to outcomes. Where sufficient information is reported, or can be supplied by the trial authors, we will re-include missing data in the analyses which we undertake. We will assess the risk of bias for incomplete outcome data as:

- low risk (e.g. no missing outcome data; missing outcome data balanced across groups; $\leq 20 \%$ missing data);

- high risk (e.g. frequency or reasons of missing data imbalanced across groups; $>20 \%$ missing data); or

- unclear risk.

\section{(5) Selective reporting (checking for reporting bias)}

We will investigate the possibility of selective outcome reporting bias by cross-checking outcomes of interest reported in the methods section to those reported in the results section of the trial publications. We will assess the risk of bias for selective reporting as:

- low risk (where it is clear that all of the study's pre-specified outcomes and all expected outcomes of interest to the review have been reported);

- high risk (where not all the study's pre-specified outcomes have been reported; one or more reported primary outcomes were not pre-specified; outcomes of interest are reported incompletely and so cannot be used; study fails to include results of a key outcome that would have been expected to have been reported); or

- unclear risk. 
(6) Other bias (checking for bias due to problems not covered by (I) to (5) above)

We will describe for each included study any important concerns we have about other possible sources of bias. For instance, given that certain biases are inherent to cluster-randomised trials due to study design, in these types of trials we will investigate potential biases due to recruitment, baseline imbalance, loss of clusters, incorrect analysis and comparability with individually-randomised trials. We will assess the risk of other forms of bias as:

- low risk;

- high risk; or

- unclear risk.

\section{(7) Overall risk of bias}

We will make explicit judgements about whether studies are at high risk of bias, according to the criteria given in the Cochrane Handbook for Systematic Reviews of Interventions (Higgins 2011). With reference to (1) to (6) above, we will assess the likely magnitude and direction of the bias and whether we consider it is likely to impact on the findings. We will explore the impact of the level of bias through undertaking sensitivity analyses (see Sensitivity analysis) and will assess the overall risk of bias for each included study as:

- low risk;

- high risk; or

- unclear risk.

\section{Measures of treatment effect}

\section{Dichotomous data}

For dichotomous data, we will present results as summary risk ratios with $95 \%$ confidence intervals $(95 \% \mathrm{CI})$.

\section{Continuous data}

For continuous data, we will use the mean difference with $95 \%$ $\mathrm{CI}$ if outcomes are measured in the same way between trials. We will use the standardised mean difference with $95 \%$ CI to combine outcomes from trials that measure the same outcome but use different scales (Higgins 2011).

\section{Unit of analysis issues}

\section{Cluster-randomised trials}

We will include cluster-randomised trials in the analyses along with individually-randomised trials. We will adjust their sample sizes using the methods described in the Cochrane Handbook for Systematic Reviews of Interventions (Higgins 2011) using an estimate of the intra-cluster correlation co-efficient (ICC) derived from the trial (if possible), from a similar trial or from a study of a similar population. If we use ICCs from other sources, we will report this and conduct sensitivity analyses to investigate the effect of variation in the ICC. If we identify both cluster-randomised trials and individually-randomised trials, we plan to synthesise the relevant information. We will consider it reasonable to combine the results from both if there is little heterogeneity between the study designs and the interaction between the effect of intervention and the choice of randomisation unit is considered to be unlikely.

We will also acknowledge heterogeneity in the randomisation unit and perform a sensitivity analysis to investigate the effects of the randomisation unit, i.e. to determine the sensitivity of the effect estimates to inclusion and exclusion of cluster trials.

\section{Multi-armed trials}

We will include multi-armed trials in this review. To overcome potential issues due to multiple, correlated comparisons, we will analyse multi-armed trials using methods described in the Cochrane Handbook for Systematic Reviews of Interventions (Higgins 2011). If feasible in the context of the research question, we will first determine if we can combine the multiple comparison groups to create one relevant intervention group and one relevant comparison group. If an appropriate pair-wise comparison cannot be created, we will then derive an average (or weighted average) and variance for all relevant intervention/comparison groups while accounting for correlation between these comparisons.

\section{Dealing with missing data}

For included studies, we will note levels of attrition. We will explore the impact of including studies with high levels of missing data (we judge this a priori to be greater than $20 \%$ for primary outcome) in the overall assessment of treatment effect by using sensitivity analysis.

For all outcomes, we will carry out analyses, as far as possible, on an intention-to-treat basis, i.e. we will attempt to include all women randomised to each group in the analyses, and all women will be analysed in the group to which they were allocated, regardless of whether or not they received the allocated intervention. The denominator for each outcome in each trial will be the number of women randomised minus any women whose outcomes are known to be missing.

\section{Assessment of heterogeneity}

Where studies are considered similar enough (based on consideration of populations and interventions) to allow pooling of data using meta-analysis, we will assess the degree of heterogeneity by visual inspection of forest plots and by examining the $\mathrm{Chi}^{2}$ test for heterogeneity. We will assess statistical heterogeneity in each meta-analysis using the $\mathrm{T}^{2}, \mathrm{I}^{2}$ and $\mathrm{Chi}^{2}$ statistics. We will regard 
statistical heterogeneity as substantial if an $\mathrm{I}^{2}$ is greater than $30 \%$ and either the $\mathrm{T}^{2}$ is greater than zero, or there is a low $\mathrm{P}$ value (less than 0.10) in the $\mathrm{Chi}^{2}$ test for heterogeneity.

Where we identify substantial clinical, methodological or statistical heterogeneity across included studies, we will not report pooled results from the meta-analysis but will instead use a narrative approach to data synthesis. In this event, we will attempt to explore possible reasons for the heterogeneity by grouping studies that have similar populations and interventions.

\section{Assessment of reporting biases}

If there are 10 or more studies in the meta-analysis, we will investigate reporting biases (such as publication bias) using funnel plots. We will assess funnel plot asymmetry visually. If asymmetry is suggested by a visual assessment, we will perform exploratory analyses to investigate it.

\section{Data synthesis}

We will carry out statistical analysis using the Review Manager software (RevMan 2011). We will use fixed-effect meta-analysis for combining data where it is reasonable to assume that studies are estimating the same underlying treatment effect: i.e. where trials are examining the same intervention, and the trials' populations and methods are judged sufficiently similar. If there is clinical heterogeneity sufficient to expect that the underlying treatment effects differ between trials, or if substantial statistical heterogeneity is detected, we will use random-effects meta-analysis to produce an overall summary if an average treatment effect across trials is considered clinically meaningful. The random-effects summary will be treated as the average range of possible treatment effects and we will discuss the clinical implications of treatment effects differing between trials. If the average treatment effect is not clinically meaningful, we will not combine trials.

If we use random-effects analyses, the results will be presented as the average treatment effect with $95 \%$ CIs and the estimates of $\mathrm{T}^{2}$ and $\mathrm{I}^{2}$

\section{Subgroup analysis and investigation of heterogeneity}

If we identify substantial heterogeneity, we will investigate this heterogeneity using subgroup analyses and sensitivity analyses. We plan to carry out the following subgroup analysis.
1. Low-risk pregnancies versus high-risk pregnancies as defined by the trial authors.

We will limit subgroup analyses to primary maternal and baby outcomes (see Types of outcome measures).

We will assess subgroup differences by interaction tests available within RevMan (RevMan 2011). We will report the results of subgroup analyses quoting the $\mathrm{Chi}^{2}$ statistic and $\mathrm{P}$ value, and the interaction test $\mathrm{I}^{2}$ value.

\section{Sensitivity analysis}

We will perform sensitivity analyses based on trial quality by repeating our analysis among only those trials judged of 'high quality'. For the purposes of this review, 'high quality' trials will be defined as trials with low risk of bias due to allocation concealment and low risk of bias due to incomplete outcome data.

We will limit sensitivity analyses to primary maternal and baby outcomes (see Types of outcome measures). Sensitivity analyses will also assist in investigating substantial statistical heterogeneity if present (see Assessment of heterogeneity).

\section{ACKNOWLEDGEMENTS}

We would like to acknowledge Lynn Hampson (Trials Search Co-ordinator), Frances Kellie (Managing Editor) and Jim Neilson (Contact Editor) of the Cochrane and Pregnancy Childbirth Group for their support throughout the drafting of this protocol.

JEL has been awarded a HRB Cochrane Review Training Fellowship from the Health Research Board (HRB), Ireland.

As part of the pre-publication editorial process, this protocol has been commented on by three peers (an editor and two referees who are external to the editorial team) and the Group's Statistical Adviser.

The National Institute for Health Research (NIHR) is the largest single funder of the Cochrane Pregnancy and Childbirth Group. The views and opinions expressed therein are those of the authors and do not necessarily reflect those of the NIHR, NHS or the Department of Health. 


\section{R E F E R E N C E S}

\section{Additional references}

\section{ACOG 2009}

ACOG Practice Bulletin No. 106. Intrapartum fetal heart rate monitoring: Nomenclature, interpretation, and general management principles. Obstetrics and Gynecology 2009; 114(1):192-202.

Alfirevic 2013

Alfirevic Z, Devane D, Gyte GML. Continuous cardiotocography (CTG) as a form of electronic fetal monitoring (EFM) for fetal assessment during labour. Cochrane Database of Systematic Reviews 2013, Issue 5. [DOI: 10.1002/14651858.CD006066.pub2]

\section{Ayres-de-Campos 2010}

Ayres-de-Campos D, Ugwumadu A, Banfield P, Lynch P, Amin P, Horwell D, et al.A randomised clinical trial of intrapartum fetal monitoring with computer analysis and alerts versus previously available monitoring. $B M C$ Pregnancy Childbirth 2010;10:71. [DOI: 10.1186/ 1471-2393-10-71]

Basciftci 2011

Basciftci F, Hatay OF. Reduced-rule based expert system by the simplification of logic functions for the diagnosis of diabetes. Computers in Biology and Medicine 2011;41(6): $350-6$.

Blix 2003

Blix E, Sviggum O, Koss KS, Oian P. Inter-observer variation in assessment of 845 labour admission tests: comparison between midwives and obstetricians in the clinical setting and two experts. BJOG: an international journal of obstetrics and gynaecology 2003;110(1):1-5.

Chauhan 2008

Chauhan SP, Klauser CK, Woodring TC, Sanderson M, Magann EF, Morrison JC. Intrapartum nonreassuring fetal heart rate tracing and prediction of adverse outcomes: interobserver variability. American Journal of Obstetrics and Gynecology 2008;199(6):623 e1-5.

\section{Chez 2011}

Chez BF, Baird SM. Electronic fetal heart rate monitoring: where are we now?. Journal of Perinatal and Neonatal Nursing 2011;25(2):180-92; quiz 93-4.

\section{Chi 2012}

Chi CL, Nick Street W, Robinson JG, Crawford MA Individualized patient-centered lifestyle recommendations: an expert system for communicating patient specific cardiovascular risk information and prioritizing lifestyle options. Journal of Biomedical Informatics 2012;45(6): 1164-74.

\section{Costa 2010}

Costa A, Santos C, Ayres-de-Campos D, Costa C, Bernardes J. Access to computerised analysis of intrapartum cardiotocographs improves clinicians' prediction of newborn umbilical artery blood $\mathrm{pH}$. BJOG: an international journal of obstetrics and gynaecology 2010;117:1288-93.

\section{Costa 2010a}

Costa MA, Ayres-de-Campos D, Machado AP, Santos C, Bernardes J. Comparison of a computer system evaluation of intrapartum cardiotocographic events and consensus of clinicians. Journal of Perinatal Medicine 2010;38:191-5.

Devane 2005

Devane D, Lalor J. Midwives' visual interpretation of intrapartum cardiotocographs: intra- and inter-observer agreement. Journal of Advanced Nursing 2005;52(2): $133-41$.

Devane 2007

Devane D, Lalor J, Bonnar J. The use of intrapartum electronic fetal heart rate monitoring: a national survey. Irish Medical Journal 2007;100(2):360-2.

Devane 2012

Devane D, Lalor JG, Daly S, McGuire W, Smith V. Cardiotocography versus intermittent auscultation of fetal heart on admission to labour ward for assessment of fetal wellbeing. Cochrane Database of Systematic Reviews 2012, Issue 2. [DOI: 10.1002/14651858.CD005122.pub4]

\section{Devoe 2000}

Devoe L, Golde S, Kilman Y, Morton D, Shea K, Waller J. A comparison of visual analyses of intrapartum fetal heart rate tracings according to the new national institute of child health and human development guidelines with computer analyses by an automated fetal heart rate monitoring system. American Journal of Obstetrics and Gynecology 2000;183(2): 361-6.

Dilenge 2001

Dilenge ME, Majnemer A, Shevell MI. Long-term developmental outcome of asphyxiated term neonates. Journal of Child Neurology 2001;16:781-92.

\section{East 2007}

East CE, Begg L, Colditz PB. Fetal pulse oximetry for fetal assessment in labour. Cochrane Database of Systematic Reviews 2007, Issue 2. Art. No.: CD004075. DOI:. [DOI: 10.1002/14651858.CD004075.pub3]

\section{East 2010}

East CE, Leader LR, Sheehan P, Henshall NE, Colditz PB. Intrapartum fetal scalp lactate sampling for fetal assessment in the presence of a non-reassuring fetal heart rate trace. Cochrane Database of Systematic Reviews 2010, Issue 3. [DOI: 10.1002/14651858.CD006174.pub2]

Fatemi 2009

Fatemi A, Wilson MA, Johnston MV. Hypoxic-ischemic encephalopathy in the term infant. Clinics in Perinatology 2009;36(4):835-58.

\section{Fawsitt 2013}

Fawsitt CG, Bourke J, Greene RA, Everard CM, Murphy A Lutomski JE. At what price? A cost-effectiveness analysis comparing trial of labour after previous caesarean versus elective repeat caesarean delivery. PLoS One 2013;8(3): e58577. DOI: 10.1371/journal.pone.0058577. 
Figueras 2005

Figueras F, Albela S, Bonino S, Palacio M, Barrau E, Hernandez $S$, et al.Visual analysis of antepartum fetal heart rate tracings: inter- and intra-observer agreement and impact of knowledge of neonatal outcome. Journal of Perinatal Medicine 2005;33(3):241-5.

Garmi 2012

Garmi G, Salim R. Epidemiology, etiology, diagnosis, and management of placenta accreta. Obstetrics and Gynecology International 2012;2012:873929. [DOI: 10.1155/2012/ 873929]

Greene 1996

Greene KR. Intelligent fetal heart rate computer systems in intrapartum surveillance. Current Opinion in Obstetrics and Gynecology 1996;8(2):123-7.

\section{Grobman 2000}

Grobman WA, Peaceman AM, Socol ML. Cost-effectiveness of elective cesarean delivery after one prior low transverse cesarean. Obstetrics and Gynecology 2000;95(5):745-51.

\section{Guijarro-Berdiñas 2002}

Guijarro-Berdiñas B, Alonso-Betanzos A, FontenlaRomero O. Intelligent analysis and pattern recognition in cardiotocographic signals using a tightly coupled hybrid system. Artificial Intelligence 2002;136:1-27.

\section{Henderson 2001}

Henderson J, McCandlish R, Kumiega L, Petrou S. Systematic review of economic aspects of alternative modes of delivery. BJOG: an international journal of obstetrics and gynaecology 2001;108(2):149-57.

Higgins 2011

Higgins JPT, Green S, editors. Cochrane Handbook for Systematic Reviews of Interventions Version 5.1.0 [updated March 2011]. The Cochrane Collaboration, 2011. Available from www.cochrane-handbook.org.

\section{Hill 2012}

Hill JB, Chauhan SP, Magann EF, Morrison JC, Abuhamad AZ. Intrapartum fetal surveillance: review of three national guidelines. American Journal of Perinatology 2012;29(7): 539-50.

Issac Niwas 2012

Issac Niwas S, Palanisamy P, Chibbar R, Zhang WJ. An expert support system for breast cancer diagnosis using color wavelet features. Journal of Medical Systems 2012;36 (5):3091-102

Liao 2005

Liao SH. Expert system methodologies and applications a decade review from 1995 to 2004. Expert Systems with Applications 2005;28(1):93-103.

\section{Liston 2007}

Liston R, Sawchuck D, Young D, Society of Obstetrics and Gynaecologists of Canada, British Columbia Perinatal Health Program. Fetal Health Surveillance: Antepartum and intrapartum consensus guideline No. 197. Journal of Obstetrics and Gynaecology Canada: JOGC 2007;29(9 Suppl 4):S3-S56.
Lydon-Rochelle 2010

Lydon-Rochelle MT, Cahill AG, Spong CY. Birth after previous cesarean delivery: short-term maternal outcomes. Seminars in Perinatology 2010 Aug;34(4):249-57.

\section{McCartney 2000}

McCartney PR. Computer analysis of the fetal heart rate. Journal of Obstetric, Gynecologic, and Neonatal Nursing 2000; 29(5):527-36.

\section{McCartney 2011}

McCartney PR. Computer fetal heart rate pattern analysis. MCN; American Journal of Maternal Child Nursing 2011;36 (6):397.

\section{McIntyre 2013}

McIntyre S, Taitz D, Keogh J, Goldsmith S, Badawi N, Blair E. A systematic review of risk factors for cerebral palsy in children born at term in developed countries. Developmental Medicine and Child Neurology 2013;55(6): 499-508.

\section{Miller 2011a}

Miller DA. Intrapartum fetal heart rate definitions and interpretation: evolving consensus. Clinical Obstetrics and Gynecology 2011;54(1):16-21.

\section{Miller 2011b}

Miller L. Intrapartum fetal monitoring: liability and documentation. Clinical Obstetrics and Gynecology 2011;54 (1):50-5.

\section{Neilson 2012}

Neilson JP. Fetal electrocardiogram (ECG) for fetal monitoring during labour. Cochrane Database of Systematic Reviews 2012, Issue 4. [DOI: 10.1002/ 14651858.CD000116.pub3]

\section{Nelson 1996}

Nelson KB, Dambrosia JM, Ting TY, Grether JK. Uncertain value of electronic fetal monitoring in predicting cerebral palsy. New England Journal of Medicine 1996;334(10): 613-8.

\section{NICE 2007}

National Institute for Health and Clinical Excellence. CG55 Intrapartum care: Care of healthy women and their babies during childbirth. NICE Clinical Guidelines. UK 2007.

\section{Pehrson 2011}

Pehrson C, Sorensen JL, Amer-Wahlin I. Evaluation and impact of cardiotocography training programmes: a systematic review. BJOG: an international journal of obstetrics and gynaecology 2011;118(8):926-36.

\section{Picon 2012}

Picon AP, Ortega NR, Watari R, Sartor C, Sacco IC. Classification of the severity of diabetic neuropathy: a new approach taking uncertainties into account using fuzzy logic. Clinics (Sao Paulo) 2012;67(2):151-6.

\section{RANZCOG 2006}

Royal Australian and New Zealand College of Obstetricians and Gynaecologists. Clinical Guidelines: Intrapartum 
Fetal Surveillance. 2nd ed. East Melbourne, Australia: RANZCOG. 2nd Ed. 2006.

\section{RevMan 2011}

The Nordic Cochrane Centre, The Cochrane Collaboration. Review Manager (RevMan). 5.1. Copenhagen: The Nordic Cochrane Centre, The Cochrane Collaboration, 2011.

\section{Seto 2012}

Seto E, Leonard KJ, Cafazzo JA, Barnsley J, Masino C, Ross HJ. Developing healthcare rule-based expert systems: case study of a heart failure telemonitoring system. International Journal of Medical Informatics 2012;81(8):556-65.
Steer 2008

Steer PJ. Has electronic fetal heart rate monitoring made a difference?. Seminars in Fetal and Neonatal Medicine 2008; 13(1):2-7.

Yang 2012

Yang F, Tian J, Xiang Y, Zhang Z, Harrington Pde B. Near infrared spectroscopy combined with least squares support vector machines and fuzzy rule-building expert system applied to diagnosis of endometrial carcinoma. Cancer Epidemiology 2012;36(3):317-23.

* Indicates the major publication for the study

\section{A P P E N DICES}

\section{Appendix I. Search terms for Open Grey and ProQuest}

\section{Open Grey}

medical equipment AND (fetal OR foetal OR birth OR labour OR labor OR childbirth) expert systems AND (fetal OR foetal OR birth OR childbirth OR labor OR labour) cardiotocograph OR cardiotocography fetal monitoring OR foetal monitoring (We will search for each line separately and assess results of each).

\section{ProQuest Dissertations and Theses} all(fetal OR foetal OR birth OR childbirth OR obstetrics) AND all((electronic NEAR/1 monitoring OR expert NEAR/2 systems OR intelligent NEAR/2 analysis OR computer NEAR/2 analysis OR cardiotocography OR cardiotocograph))

\section{CONTRIBUTIONS OFAUTHORS}

JEL drafted the initial protocol and is guarantor for the review. DD supervised the review process, critically assessed the methodology and drafted part of the protocol. RAG and CAR provided a clinical perspective; SM provided a consumer perspective. JEL, DD, CAR, $\mathrm{SM}$ and RAG revised the protocol for intellectual content and approved its submission for publication.

\section{DECLARATIONSOF INTEREST}

None known. 
SOURCES OF SUPPORT

\section{Internal sources}

- No sources of support supplied

\section{External sources}

- The Health Research Board, Ireland.

Cochrane Review Training Fellowship 